\title{
Pharmacotherapy in Female Sexual Dysfunction: IS THERE A MAGIC PILL?
}

\section{Barbosa D', Dias-Amaral A ${ }^{1}$, Covelo V'1, Ramos B' ${ }^{1}$, Mota $\mathbf{M}^{1}$}

1Psychiatry and Mental Health Department, São joão University Hospital Centre, Porto, Portugal

OBJECTIVES: To conduct a review of the existing literature regarding the established treatments for female sexual dysfunctions (FSD) and the novel pharmacological approaches that have been studied as well its clinical effectiveness.

\begin{abstract}
BACKGROUND
Women experience sex differently from men. Women may not follow a linear model when it comes to their sexual response. By proposing a more realistic model of sexual response, Basson introduced a new concept of emotional intimacy and emphasized the influence of biopsychossocial factors in women's sexuality, allowing a more comprehensive and effective approach to female sexual dysfunction (FSD).

The scientific push towards newer and better treatment approaches for FSD is relevant since female sexual complaints are common and the prevalence of FSD is about 30\%. The Diagnostic and Statistical Manual of Mental Disorders (DSM) categorizes female sexual dysfunctions in three categories: sexual interest/arousal disorders, orgasmic disorders and pain and penetration disorders. But these three main categories are not exclusive and women often complaining of more than one dificulty. This poses a demanding challenge to the clinician/therapist when it comes to treat sexual dysfunctions or problems. Health care professsionals should retain that not all female sexual complaints are psychological and there are some pharmacological options, although they still have scarce parctical implementation. However, treatment should always be tailored to each woman's particular needs, following the principles of a multidisciplinary and holistic approach.
\end{abstract}

MATERIAL AND METHODS: We performed an online literature search using the Pubmed database platform. A total of thirty-one articles were included.

\section{RESULTS}

We present the results from a categorical standpoint by organizing the evidence collected of each treatment in regards to each diagnostic category of FSD: interest and arousal disorder, pain and penetration disorders and orgasmic disorder.

The pharmacological approaches that have been studied for female sexual interest/arousal disorder are described in table 1, highlighting the clinical benefits, safety concerns and other notes regarding drug development and evidence.

Most treatments for pain and penetration disorders include use of topical agents such as lidocaine (also used via injectable administration), estradiol, nitroglycerin and even topical solutions with amitryptiline and anticonvulsants. Antidepressants as amitriptyline and other tricyclic antidepressants, duloxetine or venlafaxine, and anticonvulsants as gabapentin, pregabalin, topiramate or lamotrigine have been used orally. However, the majority of pharmacological treatment options for genital pai and penetration disorders are not evidence-based, with some patients experiencing symptomatic relief but not complete resolution of their complaints.

In most cases, there are no pharmacological options targeting orgasm disorders. Buproprion and Sildenafil have been among the most commonly tested agents but with conflicting results.

TABLE 1 - Pharmacological tReATMentS FOR FEMALE SEXUAL INTEREST/AROUSAL DISORDER

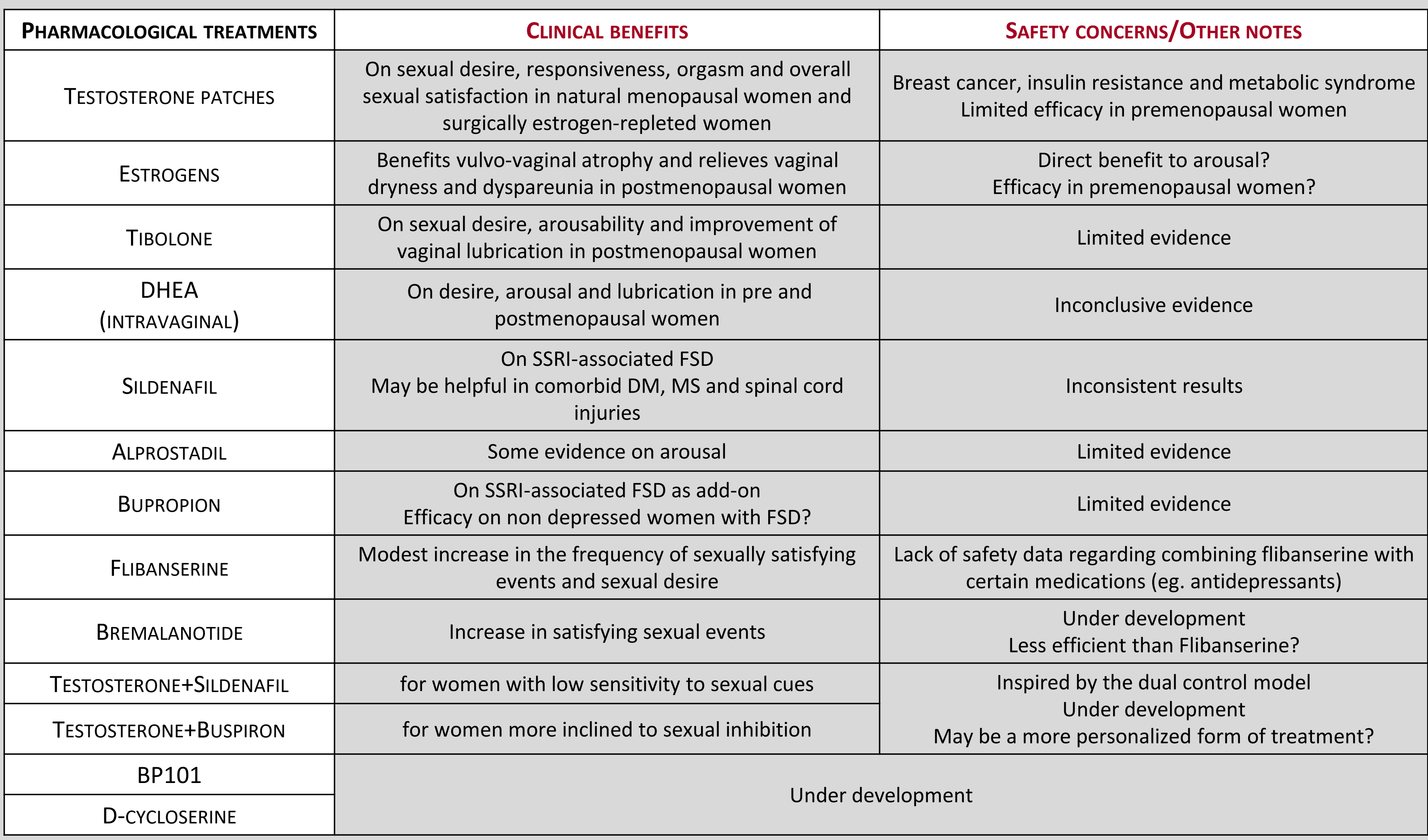

